\title{
SURFACE EQUATIONS OF STATE IN ADSORPTION FROM SOLUTION
}

\author{
ROBERT S. HANSEN and K. G. BAIKERIKAR \\ Ames Laboratory-ERDA and Department of Chemistry, Iowa State University, Ames, IA 50011, USA
}

\begin{abstract}
Evaluation of a surface equation of state generally involves an evaluation of its ability to represent raw experimental data, or information derived from such data, after the parameters of the equation have been adjusted to optimize this representation, followed by an evaluation of the physical reasonableness of the optimizing parameters in terms of the physical model on which the equation of state is based.

This process is analyzed critically for the adsorption of polar organic compounds at the mercury-electrolytic solution interface using high precision electrocapillary data as test data and the Frumkin and Flory-Huggins equations as test equations. In one approach, $\pi$ vs $\ln a$ data were represented by a set of hyperbolae with coefficients chosen to give best least squares representations of data, and adsorption isotherms obtained by analytical differentiation; parameters for the isotherms were selected to give best least squares fits to these data. Parametrizations depending on high surface pressure limiting tangents to $\pi$ vs $\ln a$ plots, intercept and slope of low surface pressure $\ln (\pi / a)$ vs $\pi$ plots, were also investigated. Parameter sets obtained by different methods of parametrization agreed only moderately well, reflecting sensitivities to different portions of the experimental data. When all Flory-Huggins parameters were freely adjusted, best fits resulted when the water co-area was taken as larger than the organic compound co-area, a physically unrealistic result.
\end{abstract}

\section{INTRODUCTION}

The Frumkin isotherm equation has been consistently employed by the Russian school of electrochemists led by Damaskin $^{1}$ in representing the adsorption of organic compounds from aqueous solution by mercury. Conway et $a l^{2-9}$ and Lawrence and Parsons ${ }^{10}$ have used a modified Flory-Huggins isotherm for interpretation of similar kinds of data. The modified Flory-Huggins isotherm is

$$
B a=\frac{\theta}{(1-\theta)^{x}} \mathrm{e}^{-2 \alpha \theta}
$$

where $a$ is the solute activity, $\theta$ the fractional surface coverage, $B, x$, and $\alpha$ are parameters. When $x=1$, eqn (1) reduces to the Frumkin equation; when $x=1$ and $\alpha=0$, eqn (1) reduces to the Langmuir equation. Physically, the parameter $B$ is related to a standard free energy of adsorption (note that $(\theta / a) \rightarrow B$ as $a \rightarrow 0$ ), $x$ is introduced in a quasilattice model of the adsorption region according to which an adsorbate molecule displaces $x$ solvent molecules, and $\alpha$ is a parameter associated with interactions between adsorbate molecules in the adsorbed layer. Conway et al. and Lawrence and Parsons have used molecular models to estimate values of $x$ in the systems they studied, thus fixing the value of $x$ a priori in eqn (1). The non-integer values of $x$ sometimes used ${ }^{10}$ are conceptually awkward in terms of the model on the basis of which eqn (1) is derived. Previous work in this laboratory ${ }^{11}$ indicates that, for polar organic molecules in water, the choice $x=1$ generally leads to a much better representation of experimental data than a value $x \approx 3$ which might be expected from size considerations.

The present work undertakes to discuss rather generally eqn (1) which (including its Langmuir and Frumkin variants) is quite widely used, and the surface van der Waals equation of state

$$
\left(\pi+\beta \theta^{2}\right)(1-\theta)=\theta \Gamma_{m} R T
$$

in which $\pi$ is the spreading pressure (boundary tension for adsorbate-free solution minus boundary tension of adsorbate solution, $\Gamma_{m}$ is the moles adsorbate $/ \mathrm{cm}^{2}$ in a complete monolayer, $R$ is the gas constant, $T$ the absolute temperature, and $\beta$ is a parameter associated with interactions between adsorbate molecules in the adsorbed layer. Equation (2) through the Gibbs adsorption equation implies the isotherm equation

$$
B a=\frac{\theta}{1-\theta} \exp \left\{\frac{\theta}{1-\theta}-\frac{2 \beta \theta}{\Gamma_{m} R T}\right\} .
$$

Similarly, eqn (1) implies the surface equation of state

$$
\frac{\pi}{\Gamma_{m} R T}=\theta-\alpha \theta^{2}-x\{\theta+\ln (1-\theta)\}
$$

Equation (3) is little used in representing adsorption from solution data. Equations (1) and (3) are attractive for comparison, however, because the first stems from a localized monolayer model, the second from a mobile monolayer model, both offer some latitude in parametrization and in this sense are rather general representatives of their respective types. Both permit two-dimensional condensation for appropriate values of the interaction parameters. Viewed as equations derived by statistical thermodynamics, both involve crude approximations in configuration counting (eqn (1)) or space exclusion (eqn (2)) and crude approximations for the energy of interaction between adsorbate molecules. The first set becomes serious in both cases as $\boldsymbol{\theta}>0.5$, and the second becomes very serious when the energy of interaction per pair times the probability that a given molecule has a partner approaches or exceeds $k T$ where $k$ is Boltzmann's constant. This will almost certainly be the case for polar organic molecules in water when $\theta>0.5$.

\section{THEORETICAL}

It is plain by inspection of eqns (1) and (3) that in either case a plot of $\theta$ against $a$ will start at the origin, have an initial slope $B$, and will approach 1 as $a$ approaches infinity no matter what values the parameters $x, \alpha$, and $\beta$ have ( $x$ is of course positive). It should be apparent that 
isotherms whose curvatures are never positive (and these are very commonly encountered) are roughly established by these statements, so they will need to be welldocumented to learn much about the other parameters.

Equations (1) and (3) are easily developed in MacLaurin series in $\theta$ to give eqns (5) and (6), respectively

$$
\begin{aligned}
B a= & \theta+(x-2 \alpha) \theta^{2}+\left\{2 \alpha(\alpha-x)+\frac{1}{2} x(x+1)\right\} \theta^{3} \\
& +\cdots \text { (Flory-Huggins) } \\
B a= & \theta+2\left(1-\frac{\beta}{\Gamma_{m} R T}\right) \theta^{2}+\left\{3-\frac{2 \beta}{\Gamma_{m} R T}\right. \\
& \left.+\frac{1}{2}\left(1-\frac{2 \beta}{\Gamma_{m} R T}\right)^{2}\right\} \theta^{3}+\cdots \text { (v.d. Waals). }
\end{aligned}
$$

Plainly the coefficients $x, \alpha$, and $\beta$ permit arbitrary adjustment of the coefficient of $\theta^{2}$ in both expansions. Hence eqns (1) and (3) can both be adjusted so that the isotherms they represent will have the same initial curvatures (as well as the same initial slopes, with $\theta \rightarrow 0$ as $a \rightarrow 0$ and $\theta \rightarrow 1$ as $a \rightarrow \infty)$. It should also be noted that the initial curvature in eqn (5) depends on $(x-2 \alpha)$, so that for any choice of $x$ a value of $\alpha$ can be chosen to give the desired curvature.

Stebbins and Halsey ${ }^{12}$ have given an interesting analysis of hard-disc monolayer isotherms. In this case, the quantity $\pi /\left(\theta \Gamma_{m} R T\right)$ can be developed in power series in $\theta$, with the coefficients through terms in $\theta^{3}$ available from rigorous theory (this of course implies coefficients through terms in $\theta^{4}$ in the expansion of $B a$ in powers of $\theta$ ). Stebbins and Halsey compare the coefficients so obtained with those obtained for the Langmuir isotherm (a variant of eqn (1), and so of eqn (5), with $x=1$ and $\alpha=0$ ) and with those obtained for the Volmer Equation (a variant of eqn (3), and so of eqn (6), with $\beta=0$ ). The coefficients for terms in $\theta, \theta^{2}$, and $\theta^{3}$ are lower than exact theory by factors of from 2 to 3 in the case of the Volmer expansion and up to 12 in the case of the Langmuir expansion. Plainly inclusion of parameters such as $x, \alpha$, and $\beta$ permits improvement of these deficiencies from the viewpoint of empirical data representation, but the analysis of Stebbins and Halsey plainly shows that the physical models underlying both eqns (1) and (3) are seriously defective.

For treatment of data at relatively low surface pressures it is convenient to consider plots of $\ln (\pi / a)$ vs $\pi$. If the double layer charge varies linearly with $\theta$ at fixed polarization, as frequently appears to be the case, then plots of this type for data taken at different polarizations should superimpose on appropriate ordinate translation. MacLaurin series representations of these plots are also readily developed to terms in $\pi^{2}$, and are respectively

$$
\begin{aligned}
& \ln \frac{\pi}{a}= \ln B \Gamma_{m} R T+\left(\alpha-\frac{1}{2} x\right) \frac{\pi}{\Gamma_{m} R T} \\
&- {\left[\left(\alpha-\frac{1}{2} x\right)^{2}+\frac{1}{6}(x+3 \alpha)^{2}\right]\left(\frac{\pi}{\Gamma_{m} R T}\right)^{2} } \\
&+ \cdots(\text { Flory-Huggins) } \\
& \ln \frac{\pi}{a}=\ln B \Gamma_{m} R T+\left(\frac{\beta}{\Gamma_{m} R T}-1\right) \frac{\pi}{\Gamma_{m} R T} \\
&+\frac{1}{2} \frac{\beta}{\Gamma_{m} R T}\left(\frac{\beta}{\Gamma_{m} R T}-2\right)\left(\frac{\pi}{\Gamma_{m} R T}\right)^{2} \\
&+\cdots \text { (v.d. Waals). }
\end{aligned}
$$

Plainly the two expansions indicate the same intercepts and the parameters $x, \alpha$, and $\beta$ can be selected to give the same initial slope. The initial slope in eqn (7) can establish $\alpha-(1 / 2) x$, but establishes neither $\alpha$ nor $x$ separately.

If the initial slope of a plot of $\ln (\pi / a)$ vs $\pi$ is positive, then the plot must have a maximum; the Gibbs adsorption theorem shows that this maximum occurs when $\theta=$ $\pi / \Gamma_{m} R T$. Equations (1) and (3) can then be used to calculate the value of $\ln (\pi / a)$ at this maximum for the Flory-Huggins and van der Waals monolayers, respectively with the results

$$
\begin{aligned}
\ln \left(\frac{\pi}{a}\right)_{\max }= & \ln B \Gamma_{m} R T+x \ln \left(1-\frac{\pi}{\Gamma_{m} R T}\right) \\
& +2 \alpha \frac{\pi}{\Gamma_{m} R T} \text { (Flory-Huggins) } \\
\ln \left(\frac{\pi}{a}\right)_{\max }= & \ln B \Gamma_{m} R T+\ln \left(1-\frac{\pi}{\Gamma_{m} R T}\right)+\frac{\pi}{\Gamma_{m} R T-\pi} \\
& -\frac{2 \beta \pi}{\left(\Gamma_{m} R T\right)^{2}}
\end{aligned}
$$$$
\text { (v.d. Waals) }
$$

Analysis of boundary tension data at high solute activities is conveniently represented by plots of $\pi$ against $\ln a$; if the double layer charge varies linearly with $\theta$ at fixed polarization, then plots of this type at different polarizations should superimpose by abscissa translation. An asymptotic representation for the Flory-Huggins isotherm valid at high surface pressures is readily found to be

$$
\pi=\Gamma_{m} R T\left[1-x+\alpha+\ln B+\ln a+x\left\{\mathrm{e}^{2 \alpha} B a\right\}^{-1 / x}\right]
$$

$$
\text { (Flory-Huggins). }
$$

This yields a well defined limiting tangent with slope $\Gamma_{m} R T$ and intercept on $\ln a$ axis $-\ln B-1+x-\alpha$ again depending on a combination of $x$ and $\alpha$; appearance of $x$ in the power dependence of the first order deviation from the limiting tangent suggests the possibility of getting at $x$ directly in this way, but the range where a single correction suffices is sufficiently small that this approach is not very promising. The corresponding asymptotic form for the van der Waals equation leads to

$$
\pi=\Gamma_{m} R T\left[\frac{\beta}{\Gamma_{m} R T}+\ln B+\ln a+\ln u+\frac{u}{\Gamma_{m} R T}+\cdots\right]
$$

(van der Waals)

where to sufficient approximation $u=[\ln \{B a / \ln B a\}]^{-1}$ The appearance of the term in $\ln u$ obscures the limiting tangent; the limiting slope is plainly $\Gamma_{m} R T$ but the tangent is ill-defined. Comparison of eqns (1) and (3), however, should indicate that, no matter what parameters are chosen, the activity given by eqn (3) will exceed that given by eqn (1) for $\theta$ sufficiently close to 1 . A lattice model will in general be favored over a mobile model as full coverage is approached. This point has been emphasized by Stebbins and Halsey ${ }^{12}$ in their comparison of the Langmuir and Volmer equations, and they also analyzed possible phase transitions between mobile and localized monolayers. It is, therefore, unlikely that eqn (12) will prove useful, for it is unlikely that mobile monolayers will exist at values of $\boldsymbol{\theta}$ approaching full coverage. 
RESULTS AND DISCUSSIONS

Details of apparatus, experimental procedures, and electrocapillary data analysis have been reported previously. ${ }^{11,13,14}$ The present experimental work concerns the adsorption of butanol-1, isopentanol (3-methyl butanol-1), $n$-pentanoic acid and $n$-hexanoic acid.

Figure 1 shows a composite $\pi$ vs $\ln a$ plot representing the adsorption of butanol- 1 at the mercury-electrolytic solution interface at $25^{\circ} \mathrm{C}$. The base solution is $0.1 \mathrm{~N}$ aqueous perchloric acid. Butanol-1 and the other organic solutes used in the present work are all of limited solubility in water and their activities were taken equal to their concentrations divided by their saturation concent-

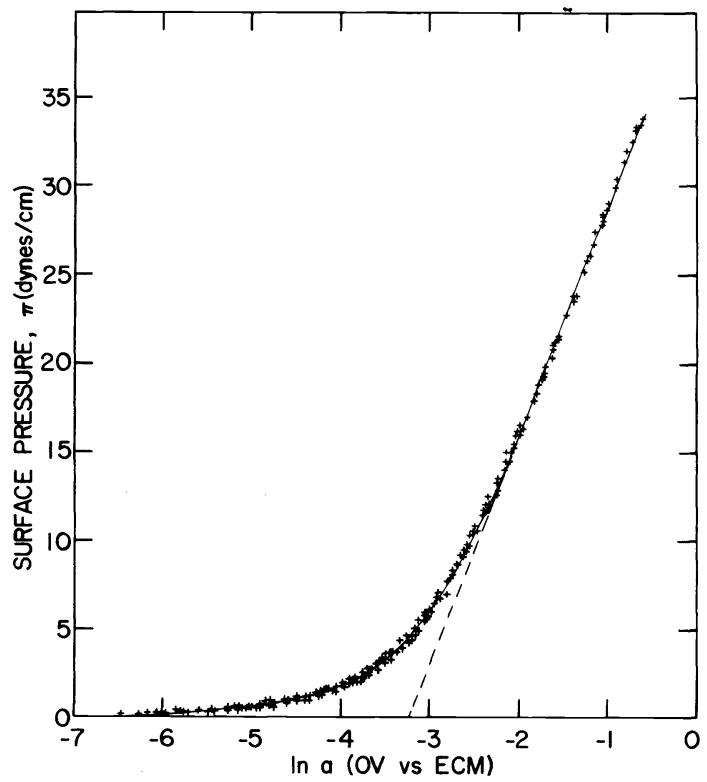

Fig. 1. Composite $\pi$ vs $\ln a$ plot for butanol-1. Points shown are experimental data; the curve is least squares fit to the data with a linear combination of hyperbolae. The RMS deviation of the points from the curve is $0.18 \mathrm{dyn} / \mathrm{cm}$. The limiting tangent (dashed line) drawn through the monolayer region is also shown. rations in the base electrolyte solution. This approximation could possibly affect conclusions of the present work only to the extent that solute activity coefficients varied appreciably over the experimental concentration range. This range extended from zero to half saturation concentration.

The data in Fig. 1 are derived from 11 electrocapillary curves (base electrolyte and 10 different solute concentrations) each documented with points at $50 \mathrm{mV}$ polarization intervals. Twenty-five constant polarization $\pi$ vs $\ln a$ plots were then made (each with 10 points) and their abscissas translated to give best superposition, with a plot at the electrocapillary maximum taken as reference. A linear combination of hyperbolas was chosen, using a computer program, to best fit the 250 points shown in Fig. 1. The solid curve shown is the analytic representation of the data thus obtained, and represents the data with an RMS deviation of $0.18 \mathrm{dyn} / \mathrm{cm}$. Data for other systems were similarly treated with similar RMS deviations, indicating that in these systems $\pi$ vs $\ln a$ plots at different polarizations are indeed superimposable by abscissa translation and that therefore, within the limits of sensitivity of the superposition test, the double layer charge must vary linearly with $\theta$ at fixed polarization and the parameters $x, \alpha$, and $\beta$ must be independent of polarization in these systems

Figure 1 also shows the limiting tangent (which of course also corresponds to the asymptote of the analytic representation of the data). The slope of this tangent was used to obtain $\Gamma_{m}$ for all further data analysis in the butanol-1 system, and the intercept provided an estimate of the Flory-Huggins parameter group $\ln B+\alpha+1-x$ [see discussion following eqn (11)].

The best analytic representation (linear combination of hyperbolas) of the data shown in Fig. 1 was then differentiated analytically to obtain $\ln a$ as a function of $\theta$, and parameters $B, x$, and $\alpha$ in the Flory-Huggins equation, eqn (1), selected through a computer program to best fit the ln $\boldsymbol{a}$ vs $\boldsymbol{\theta}$ data derived from experiment. Check calculations showed that similar parameters resulted when $\pi$ vs $\theta$ data were used with eqn (4) as test equation. Data for other solutes were similarly treated.

Figure 2 presents $\ln (\pi / a)$ vs $\pi$ data with butanol-1 as

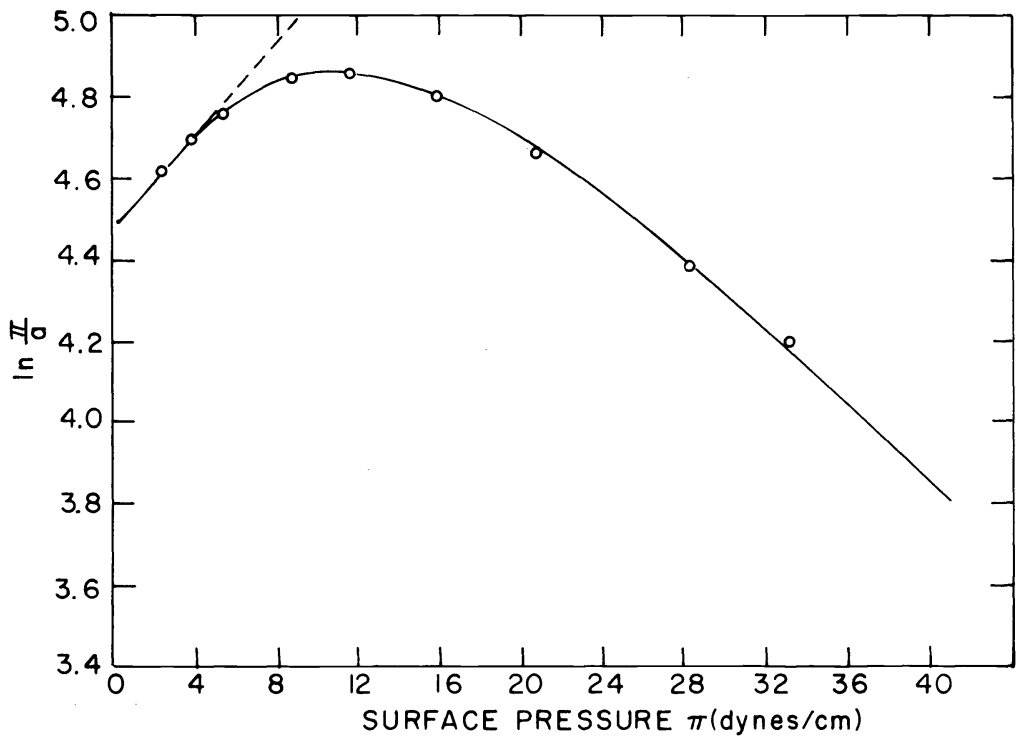

Fig. 2. In ( $\pi / a)$ vs $\pi$ plot for butanol-1 at uncharged mercury- $0.1 \mathrm{~N} \mathrm{HClO}_{4}$ solution interface. The limiting tangent (dashed line) drawn through the low surface pressure region is also shown. 
adsorbate, showing the low surface pressure limiting tangent. The intercept on the $\pi=0$ axis is $\ln B \Gamma_{m} R T$ for either the Flory-Huggins or van der Waals model (see eqns (7) and (8)), and since $\Gamma_{m} R T$ is known the intercept provides an estimate of the parameter $B$. The slope of the initial tangent is $(\alpha-(1 / 2) x) / \Gamma_{m} R T$ or $\left(\beta / \Gamma_{m} R T\right)-1$, providing estimates of the parameter group $\alpha-(1 / 2) x$ in the Flory-Huggins model and of the parameter $\beta$ in the van der Waals model.

Figure 3 shows similar plots at various polarizations based on data with $n$-pentanoic acid as adsorbate. The resemblance to Fig. 2 is plain and Fig. 3 also makes plain that curves at different polarizations are related by simple ordinate translation. It can also be seen that scatter of data at low values of $\pi$ may lead to uncertainty in location of the initial tangent. An alternate source of an equation relating parameters lies in the plot maximum as indicated by eqns (9) and (10). If the plot intercepts are sufficiently well defined to establish $\ln B \Gamma_{m} R T$ then eqn (10) establishes $\beta$ and eqn (9) furnishes a relation between the two parameters $\alpha$ and $x$.

Table 1 shows parameterizations of the Flory-Huggins representations of the four systems according to several schemes. In all schemes the limiting tangents to $\pi$ - $\ln a$ plots such as Fig. 1 are used to establish $\Gamma_{m}$.

In the first method, analytical representations of data such as shown in Fig. 1 were differentiated analytically to obtain $\ln a$ vs $\theta$ curves, and Flory-Huggins parameters were selected by a computer program to obtain the least mean square deviation from the $\ln a$ vs $\theta$ data.

In both the second and third method, the limiting tangent intercepts in the $\pi$ vs $\ln a$ plots such as Fig. 1 were used to establish the sum of parameters (In $B+1-x+\alpha)$, and the intercepts $\pi=0$ of plots such as Fig. 2 were used to establish $\ln B$. Hence both methods 2 and 3 lead to the same values of the parameter $B$ and the parameter sum $(1-x+\alpha)$. If $x$ is fixed at 1 (Frumkin isotherm), this information suffices to establish all other parameters uniquely.

In method 2, the slope of the initial tangent in plots such as Fig. 2 is used to establish $(\alpha-(1 / 2) x)$ as explained following eqn (8); since $1-x+\alpha$ is also known $\alpha$ and $x$
Table 1. Parameters of the Flory-Huggins equation for several organic solutes with size factor $x$ varied for best fit and fixed at 1 (Frumkin equation)

\begin{tabular}{|c|c|c|c|c|c|}
\hline Solute & Method & $B$ & $x$ & $\alpha$ & $\alpha^{\prime}(x=1)$ \\
\hline Butanol-1 & (1) & 7.77 & 0.43 & 0.62 & \\
\hline $10^{10} \Gamma_{m}=$ & (2) & 6.93 & 0.64 & 0.98 & $1.34 \dagger$ \\
\hline $5.19 \mathrm{~mol} / \mathrm{cm}^{2}$ & (3) & 6.93 & 0.95 & 1.29 & $1.35 \ddagger$ \\
\hline Isopentanol & (1) & 5.59 & 0.46 & 0.95 & \\
\hline $10^{10} \Gamma_{m}=$ & (2) & 5.11 & 1.14 & 1.76 & $1.62 \dagger$ \\
\hline $4.76 \mathrm{~mol} / \mathrm{cm}^{2}$ & (3) & 5.11 & 1.12 & 1.74 & $1.59 \ddagger$ \\
\hline$n$-Pentanoic acid & (1) & 8.59 & 0.44 & 0.60 & \\
\hline $10^{10} \Gamma_{m}=$ & (2) & 9.11 & & & $1.07 \dagger$ \\
\hline $4.80 \mathrm{~mol} / \mathrm{cm}^{2}$ & (3) & 9.11 & $(0.85)$ & $(0.92)$ & $1.06 \ddagger$ \\
\hline$n$-Hexanoic acid & (1) & 8.57 & 0.59 & 0.96 & \\
\hline $10^{10} \Gamma_{m}=$ & (2) & 7.63 & & & $1.29 \dagger$ \\
\hline $4.20 \mathrm{~mol} / \mathrm{cm}^{2}$ & (3) & 7.63 & 1.30 & 1.59 & $1.34 \ddagger$ \\
\hline
\end{tabular}

$\Gamma_{m}$ for all methods based on limiting slopes of $\pi$ vs $\ln a$ data as shown in Fig. (1)

Method 1: Parameters $B, x$, and $\alpha$ selected for least mean square deviation from $\ln a$ vs $\theta$ data using computer program.

Method 2: Based on intercept of $\pi$ vs $\ln a$ limiting tangent (Fig. (1)) and intercept and slope of initial tangent to $\ln (\pi / a)$ vs $\pi$ plot (Fig. (2)).

tdenotes omission of initial tangent slope.

Method 3: Based on intercept of $\pi$ vs $\ln a$ limiting tangent (Fig. (1)), intercept of initial tangent to $\ln (\pi / a)$ vs $\pi$ plot (Fig. (2)) and maximum in this latter plot.

łdenotes omission of limiting tangent intercept (Fig. (1)).

can be determined independently. In method 3 , the additional relation between $x$ and $\alpha$ is obtained from the maxima in plots such as Fig. 2, as indicated in eqn (9). If $x$ is fixed at 1 , the parameters $\ln B$ and $\alpha$ can be also obtained by considering only the intercept and maximum in $\ln (\pi / a)$ vs $\pi$ plots, without using the intercept of the limiting tangent to the $\pi$ vs $\ln a$ data.

The different methods of parameterization lead to moderate differences in the parameters selected, reflecting in part different regions of the surface pressure-

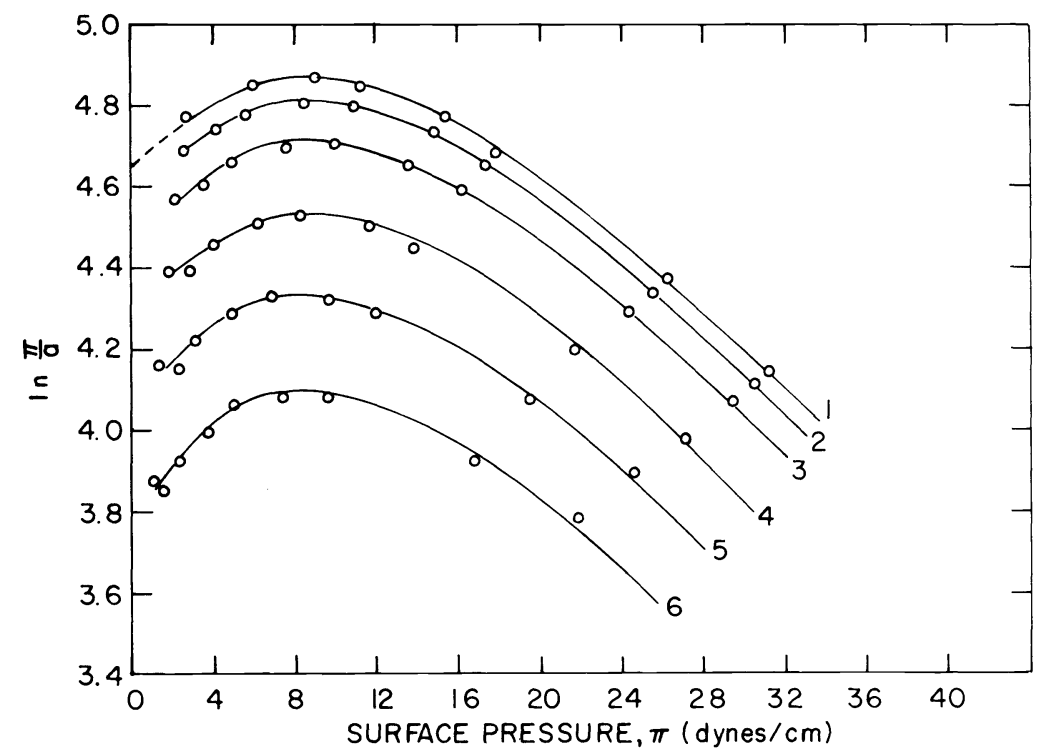

Fig. 3. $\ln (\pi / a)$ vs $\pi$ plots for $n$-pentanoic acid at the following potentials: (1)0.000V, (2) $-0.150 \mathrm{~V},(3)-0.200 \mathrm{~V}$, (4) $0.150 \mathrm{~V},(5) 0.200 \mathrm{~V}$, and (6) $0.250 \mathrm{~V}$. All potentials are in volt vs $\mathrm{ECM}$ of mercury in $0.1 \mathrm{~N} \mathrm{HClO}_{4}$ solution. 
activity data emphasized in the treatment. All three methods lead to very good representations of data at high activities; the computer parameterization emphasizes in addition data at intermediate activities, while the two methods based on $\ln (\pi / a)$ vs $\pi$ plots emphasize data at low activities. Values of $x$ obtained were systematically about 0.5 with the computer parametrization, about 1.0 by the other two methods. These values are far below the value of about 3 which might be expected from size considerations, and fits obtained with $x=3, B$ and $\alpha$ optimized for this choice of $x$, were much less satisfactory than similar fits starting with $x=1$ (which in turn were somewhat less satisfactory than those obtained with the parameters listed in Table 1).

The van der Waals equation, eqn (2), proved quite satisfactory for fitting data in the range $0 \leq \theta \leq 0.5$, with values of $\theta$ based on the $\Gamma_{m}$ values listed in Table 1. A substantial extension in range (to about $\theta=0.8$ ) could be achieved by replacing the term $(1-\theta)$ on the left side of eqn (2) with the term $(1-b \theta)$, with $b<1$. This is equivalent to using a higher value of $\Gamma_{m}$ than that obtained from the limiting slope of the $\pi$ vs $\ln a$ plots as given in Table 1, or a smaller co-area (e.g. $21 \AA^{2}$ instead of $32 \AA^{2}$ in the case of butanol-1). Even with the additional parameter, it appeared impossible to choose parameters in the van der Waals equation to represent data for $\pi>$ $15 \mathrm{dyn} / \mathrm{cm}$. The argument of Stebbins and Halsey ${ }^{12}$ as to the instability of a mobile monolayer with respect to transition to a lattice monolayer at sufficiently high activity is consistent with this finding.

\section{REFERENCES}

${ }^{1}$ B. B. Damaskin, Adsorption of Organic Compounds on Electrodes (edited by B. B. Damaskin, O. A. Petrii and V. V. Batrakov), Plenum Press, New York (1971).

${ }^{2}$ B. E. Conway and L. G. M. Gordon, J. Phys. Chem. 73, 3609 (1969).

${ }^{3}$ B. E. Conway, H. P. Dhar and S. Gottesfeld, J. Colloid Interface Sci. 43303 (1973).

${ }^{4}$ H. P. Dhar, B. E. Conway and K. M. Joshi, Electrochim. Acta 18, 789 (1973).

${ }^{5}$ B. E. Conway and H. P. Dhar, Surface Sci. 44, 261 (1974).

${ }^{6}$ B. E. Conway and H. P. Dhar, J. Colloid Interface Sci. 48, 73 (1974).

${ }^{7}$ B. E. Conway and H. P. Dhar, Electrochim. Acta 19, 445 (1974).

${ }^{8}$ B. E. Conway, H. Angerstein-Kozlowska and H. P. Dhar, Electrochim. Acta 19, 455 (1974).

${ }^{9}$ B. E. Conway, J. G. Mathieson and H. P. Dhar, J. Phys. Chem. 78, 1226 (1974).

${ }^{10}$ J. Lawrence and R. Parsons, J. Phys. Chem. 73, 3577 (1969).

${ }^{11}$ K. G. Baikerikar and R. S. Hansen, Surface Sci. 50, 527 (1975).

${ }^{12}$ J. P. Stebbins and G. D. Halsey, Jr., J. Phys. Chem. 68, 3863 (1964).

${ }^{13}$ D. E. Broadhead, R. S. Hansen and G. W. Potter, J. Colloid Interface Sci. 31, 61 (1969).

${ }^{14}$ K. G. Baikerikar and R. S. Hansen, J. Colloid Interface Sci. 52, 277 (1975). 